\title{
Using a Haptic Device and Virtual Sculpting Software For Predicting a Missing Mandible: the Case of Angelo Poliziano
}

\author{
G. Mahoney ${ }^{1}$, C. Milani ${ }^{2}$, M. Billinger ${ }^{3}$, V. Lywood ${ }^{4}$, G. Gruppioni ${ }^{5}$ \\ ${ }^{1}$ Forensic Artist, Boston Police Department, USA \\ ${ }^{2}$ DDS, Forensic Odontologist, Torino, IT. E-mail: milanich@gmail.com \\ ${ }^{3} \mathrm{PhD}$., Michael Billinger Consulting Services, Ottawa, CA \\ ${ }^{4}$ Forensic Artist, John Abbot Collage, Montreal, CA \\ ${ }^{5}$ Dep. of Conservation of Cultural Heritage, University of Bologna (Ravenna), Italy
}

KEY WORDS: forensic, anthropology, virtual, reconstruction.

\section{Introduction}

A "haptic device" and virtual sculpting has been utilized to predict the size and shape of a missing mandible in order to complete the skull and the related facial approximation of the 15 th century humanist, Angelo Poliziano. A reconstruction of the skull from fragmentary craniofacial remains was achieved in a previous project using geometric morphometric techniques (Benazzi et al., 2009). The results of this project formed the basis for subsequent modeling of the face of Poliziano. Incorporating the Sassouni method of cephalometric analysis (Sassouni, 1957), this software/hardware system allows for precise measurements combined with flexibility and speed within a $3 D$ virtual workspace in which the artist is limited only by the prediction method used. While there is an inherent subjectivity to any facial approximation, our results demonstrate that using this approach greatly reduces subjectivity. Forensic anthropologists and artists are often confronted with incomplete human remains from which to work with, posing additional challenges to an already difficult task of approximating what the face of a victim might have looked like ante mortem in order to make a positive identification. Prediction of morphology of the mandible is particularly difficult where only fragments have been found, or the mandible is missing altogether.

\section{Material and Methods}

The remains of Angelo Poliziano, which were exhumed in 2007 in Florence, were extremely fragmented and incomplete. They were captured by computed tomography (CT) scans. After a long and complex process of digital reconstruction based on morphometric geometrics (Benazzi et al., 2009), it was possible to reassemble them and to build a complete craniofacial model with the aid of a compatible reference skull including an intact lower jaw that was not affected by any deformation. The problem, however, was that it was only possible to get a general sense of the appearance of Poliziano, since the model was highly influenced by the characteristics of the reference skull. The reconstruction proceeded based on the previously constructed model without the use of the mandible associated to the reference skull. A 3D stereolithography (.STL) file of the skull obtained in the aforemention project was imported into the software program, Freeform Modeling Plus v.11.0, which incorporates a haptic device to allow an operator to manipulate a 3D file with the feeling of touch. This is often referred to as virtual sculpture. The STL file of a fragment of the left ascending ramus, including the condyle and coming from the original Poliziano remains, was used to predict the morphology of the complete ascending ramus, superimposing it in a new compatible mandible used as a reference. A mirror image of the fragment was then used to complete the right ascending ramus.

Step 1:The skull without the lower jaw was oriented on the Frankfort horizontal (Fig. 1).

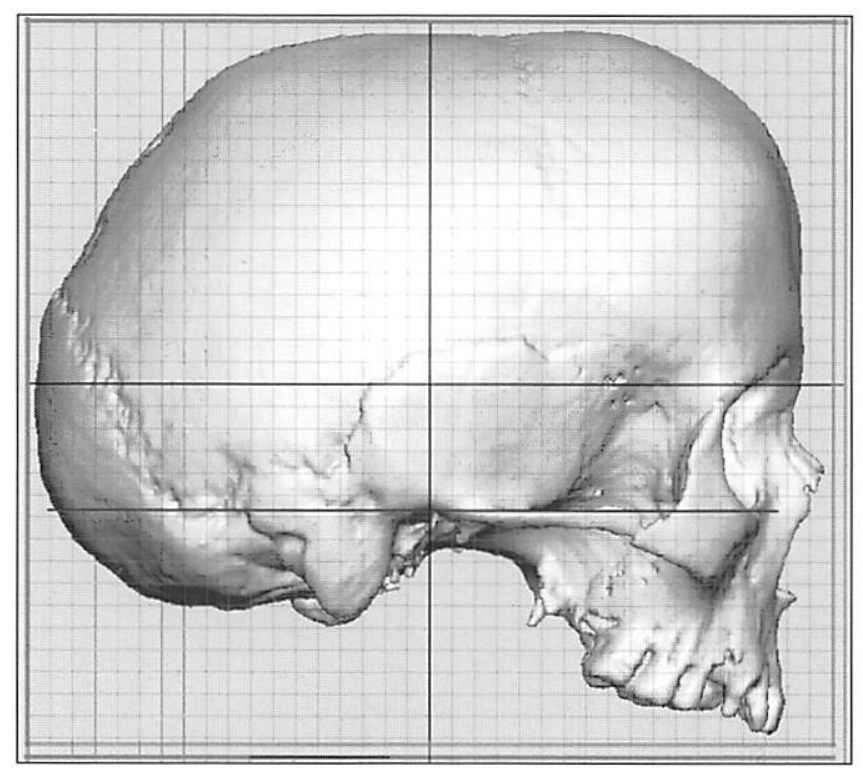

Fig. 1. Skull oriented on the Frankfort plane.

Step 2:The reconstructed lower jaw was imported and moved into proper anatomical position in order to begin the prediction of the mandibular body. Using the haptic 
device (Fig. 2) it was possible to precisely align the condyles with the articular surface of the glenoid fossa. (Fig. 3).

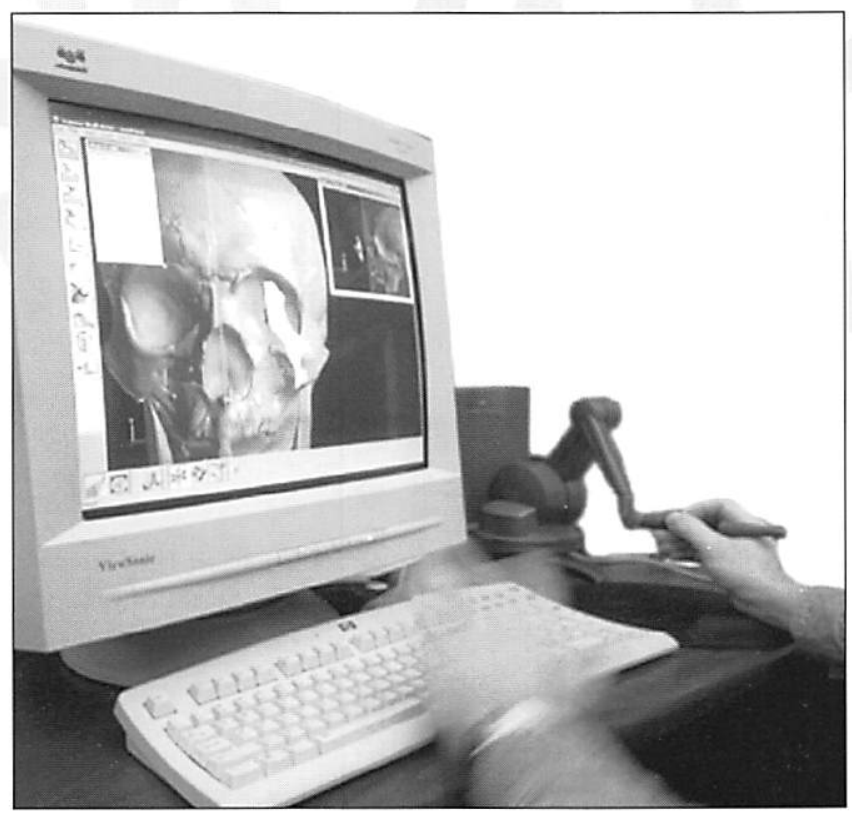

Fig. 2. Haptic device.

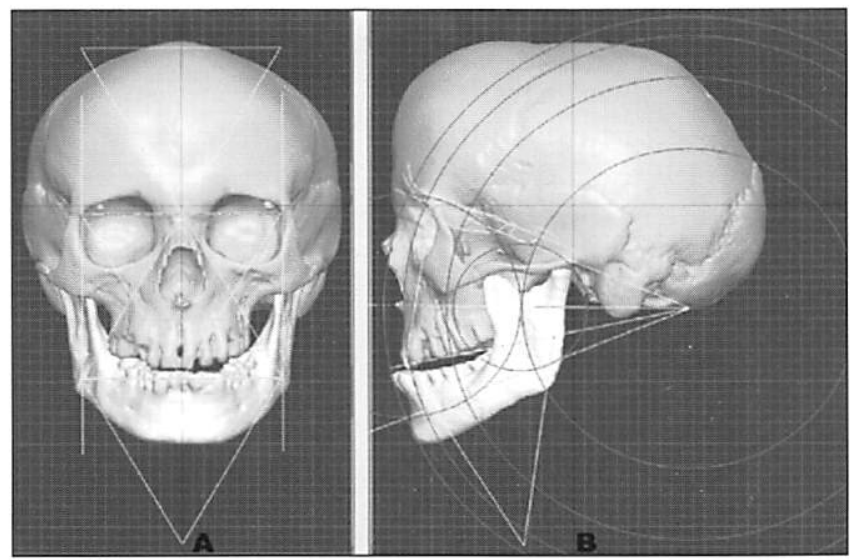

Fig. 3. Placement of the mandible, frontal and lateral view.

Step 3:The inferior jaw, now correctly positioned, were deformed according to the rules described by Sassouni (1957), which describes the proportions that a mandible should have according to angles and distances of various elements of the skull.We proceeded with the identification of the landmarks necessary for the analysis and the tracing of the horizontal planes described by the Sassouni method (Krogman et al. 1986; Sassouni, 1957, 1963), (anterior cranial base, bispinale, occlusal, mandibular). These planes, in a well proportioned face, should converge at a point called O. (Fig. 4, pink lines) The more or less rapid convergence of these plans reflect the vertical proportions of the face and the resulting malocclusion.

Step 4: Once the point of convergence has been determined, on the sagittal plane, arcs were traced with centre point $O$ passing through various points of reference, used to determine the craniofacial proportions. (Fig. 4, blue lines) The anterior nasal spine, upper teeth and the chin should lie on the same arc.

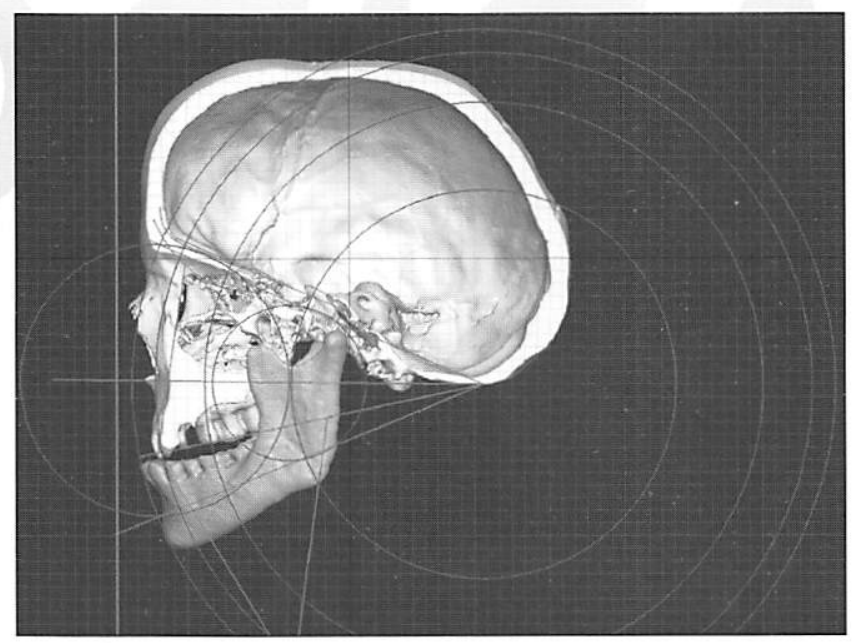

Fig. 4. Planes and arcs according with Sassouni's cephalometric analysis.

Step 5:The mandible was then deformed on the basis of points expected from the above analysis. (Fig. 5).

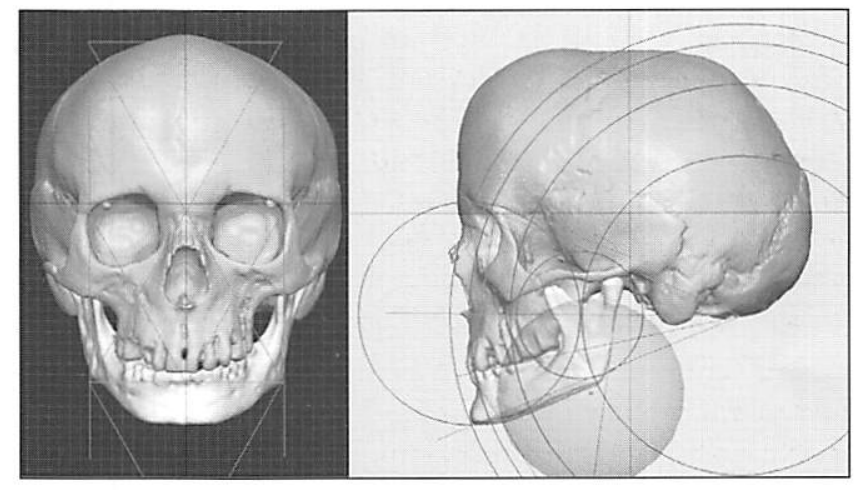

Fig.5. Mandible after Sassouni deformation, frontal and lateral view.

\section{Conclusions}

Working in three dimensions is a more complex task than it might seem, since the 3D virtual space is always displayed on a $2 \mathrm{D}$ monitor. The haptic device is extremely useful in this case: offering greater or lesser resistance according to the space in which we move and the bony structures reached virtually by the pointer, which allows for greater accuracy.

Utilizing the Sassouni method of cephalometric prediction, we were able to adjust the jaw to the preexisting cranial model so that it became compatible, incorporating morphological indicators from existing fragments of the mandible.

From the first observation, it was noted that the mandible obtained by the technique was particularly weak, so the forensic artist (unaware from the outset of the identity of the human remains and physical features of Poliziano) needed to adjust the proportions according to his considerable experience and training (Fig. 6).

We can not exclude, however, that the original mandible (Fig. 5) obtained using the Sassouni method most closely approximates to that of Angelo Poliziano. 
G. Mahoney, C. Milani, M. Billinger,V. Lywood, G. Gruppioni

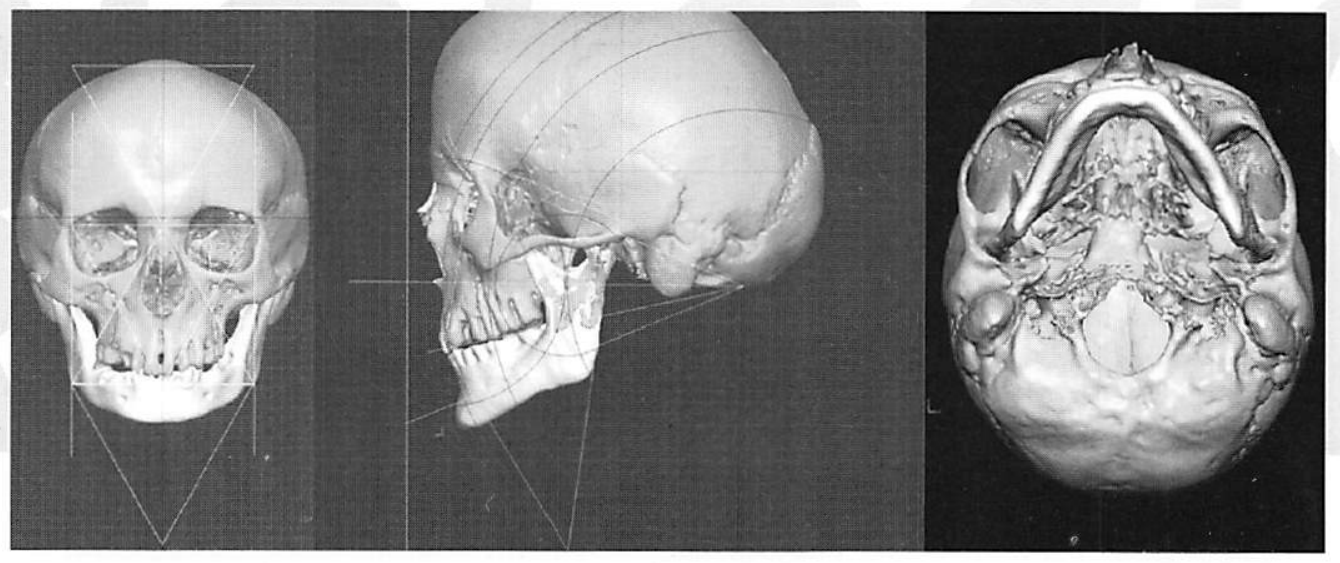

Fig.6. Final mandible.

\section{References}

Benazzi S., Stansfield E., Milani C., Gruppioni G. 2009. Geometric morphometric methods for three-dimensional virtual reconstruction of a fragmented cranium: the case of Angelo Poliziano. Int.J. Legal Medicine, 123: 333-344.

Sassouni V. 1957. Palatoprint and Roentgenographic Cephalometry as new method in Human identification. J. Forensic Sci. 2(4): 428-442.
Sassouni V. 1963. Dentofacial Radiology in Forensic Dentisty. J. Dent. Res., 42: 274-302.

Krogman W.M., Iscan M.Y. 1986. Radiographic Analysis. The Human Skeleton In Forensic Medicine. 2nd ed. United States of America, Springfield: 458 - 479. 